\title{
The effect of central shielding in the dose reporting for cervical cancer in EQD2 era
}

\author{
Ekkasit Tharavichitkul, MD', Somsak Wanwilairat, PhD', Anirut Watcharawipha, MScl, Damrongsak Tippanya, BScl, \\ Rungtip Jayasvasti, BSc', Somvilai Chakrabandhu, MD!', Pitchayaponne Klunklin, MD!' Wimrak Onchan, MD!' \\ Razvan Galalae, MD, PhD², Imjai Chitapanarux, MD \\ IThe Division of Therapeutic Radiology and Oncology, Department of Radiology, Faculty of Medicine, Chiang Mai University, Chiang Mai, \\ Thailand, 2Faculty of Medicine, Christian-Albrechts-University, Kiel, Germany
}

\begin{abstract}
Purpose: To evaluate the cumulative dose at point A for three and four centimeters central shielding.

Material and methods: The plans of external beam radiotherapy plus conventional intracavitary brachytherapy were performed. Three or four centimeters central shieldings (after $44 \mathrm{~Gy}$ ) were applied to the standard whole pelvis irradiation. Additional intracavitary brachytherapy $4 \times 7$ Gy at point A was prescribed, and the cumulative dose in EQD2 $(\alpha / \beta=10)$ of $3 \mathrm{~cm}$ and $4 \mathrm{~cm}$ central shielding were evaluated.

Results: The cumulative dose at point A in EQD2 $(\alpha / \beta=10)$ of $3 \mathrm{~cm}$ central shielding were 95.7 Gy for AR and 95.5 Gy for AL, while the cumulative dose at point As in EQD2 $(\alpha / \beta=10)$ of $4 \mathrm{~cm}$ central shielding were $90.8 \mathrm{~Gy}$ for AR and 91.2 Gy for AL.

Conclusions: The $3 \mathrm{~cm}$ central shielding caused higher cumulative dose (in terms of EQD2 $[\alpha / \beta=10]$ ) than $4 \mathrm{~cm}$ central shielding.
\end{abstract}

Key words: central shielding, cervical cancer, EQD2.

\section{Purpose}

Cervical cancer is one of the most common cancers in Thai people. In Northern Thailand, the incidence of cervical cancer is 23.5 per 100000 [1]. Radiation therapy (with chemotherapy) plays a major role for treatment of locally advanced cervical cancer, and the combination of whole pelvic radiotherapy (WPRT) plus intracavitary brachytherapy (ICBT) are used to treat them [2-4]. The goal of WPRT is to treat primary tumors/pelvic lymph-nodes with the goal of decrease tumor size. Brachytherapy is used to escalate the dose for curative outcomes. In our institution, conventional radiation therapy (2D treatment) has been used, and whole pelvic radiotherapy to the dose of 56 Gy with central shielding (after $44 \mathrm{~Gy}$ ) plus intracavitary brachytherapy with $4 \times 7$ Gy at point A was used with promising results [5]. The $3-\mathrm{cm}$ or $4-\mathrm{cm}$ central shielding were used to reduce the dose for bladder and rectum. Normally, our institute use 4-cm central shielding as standard shielding. However, there are some limitations to use $4-\mathrm{cm}$ central shielding in case of modified segmental boost technique (to treat whole pelvis plus inguinal area) for stage IIIA (lower vaginal involvement). In this case, the 3-cm central shielding will be used. With the concept of equivalent dose of $2 \mathrm{~Gy}$ (EQD2), the cumulative dose to the tumor can be calculated [6]. The cumulative EQD2 in each type of central shielding is interesting to our institution, regarding the question of dose reporting. According to our practice in the institute, we performed this test to evaluate the cumulative dose in EQD2 concepts at point A, when $3-\mathrm{cm}$ and $4-\mathrm{cm}$ central shieldings were used after $44 \mathrm{~Gy}$.

\section{Material and methods}

To identify this calculation, WPRT was used with a dose of $2 \mathrm{~Gy} /$ fraction, 5 fractions per week to a dose of $44 \mathrm{~Gy}$ in AP-PA technique. After $44 \mathrm{~Gy}$, the central shielding was added to the dose of 50 Gy with conventional fractionation in an AP/PA technique. Then a parametrial boost with the same size of central shielding was used with an additional dose of 6 Gy with 2 Gy per fraction to the total dose of $56 \mathrm{~Gy}$. All phases were planned in a Pinnacle $^{\mathrm{TM}}$ treatment planning system (Royal Philips, Amsterdam, The Netherlands). To evaluate our question, two types of central shielding were added to this field after $44 \mathrm{~Gy}$. The plan was performed on CT images for $2 \mathrm{~Gy}$ 
per fraction. The point $\mathrm{A}$ and point $\mathrm{A}-5 \mathrm{~mm}$ which represented the point at the borders of $4-\mathrm{cm}$ and $3-\mathrm{cm}$ central shielding were supposedly added into the WPRT, and the doses at these points were recorded in each type of central shielding. The total doses of WPRT were evaluated and recorded in each setting (Fig. 1).

For ICBT, an AP film with a good geometry of application (the tandem is centrally located in the field) was used. Point AR and AL were added into the film for digitization into the Plato coordinate system. The point AR-5mm and AL-5mm were also added into the coordinate system (Fig. 2). The prescription dose of $4 \times 7$ Gy to point A was prescribed and calculated in EQD2 concepts $(\alpha / \beta=10 \mathrm{~Gy})$. The cumulative EQD2 $(\alpha / \beta=10 \mathrm{~Gy})$ doses of WPRT plus ICBT for each type of central shielding were evaluated.

\section{Results}

\section{For whole pelvic radiotherapy}

The physical dose at point $\mathrm{AR}, \mathrm{AL}, \mathrm{AR}-5 \mathrm{~mm}$, and AL-5mm are shown in Table 1 , and the cumulative doses in EQD2 $(\alpha / \beta=10)$ for AR, AL, AR-5mm, and AL-5mm are presented in Table 2 .

\section{For intracavitary brachytherapy}

The dose at point A-5mm was $140 \%$ of the dose at point $A$. The cumulative dose for four times of ICBT in point A- $5 \mathrm{~mm}$ and point A were 64.7 Gy and 39.7 Gy in EQD2 $(\alpha / \beta=10)$ concepts, respectively.

\section{In combination dose in EQD2}

The cumulative dose in EQD2 $(\alpha / \beta=10)$ concepts at point A of 3-cm central shielding had approximately 4 Gy difference from 4-cm central shielding. All data are shown in Table 3.

\section{Discussion}

From the conventional planning, after 30-46 Gy for locally advanced disease, central shielding (CS) or midline block (MLB) was applied and simple rectangular shielding has been used in routine practice. Many guidelines reported the schedules of combined doses of WPRT plus BT for locally advanced cervical cancer. The American Brachytherapy Society reported the schedule of WPRT plus BT with the dose of 45-50.4 Gy in 25-28 fractions for WPRT (with 4-cm or 5-cm wide of central shielding) [7]. A publication from Japan reported a schedule for locally advanced disease of 45-50.4 Gy in 25-28 fractions for WPRT (with central shielding after $30 \mathrm{~Gy}$ ) [8]. However, both publications did not describe the effect of size of shielding in cumulative dose calculation. In our study, the width of the shielding causes a different cumulative dose in EQD2 $(\alpha / \beta=10)$. With the $3 \mathrm{~cm}$ central shielding,
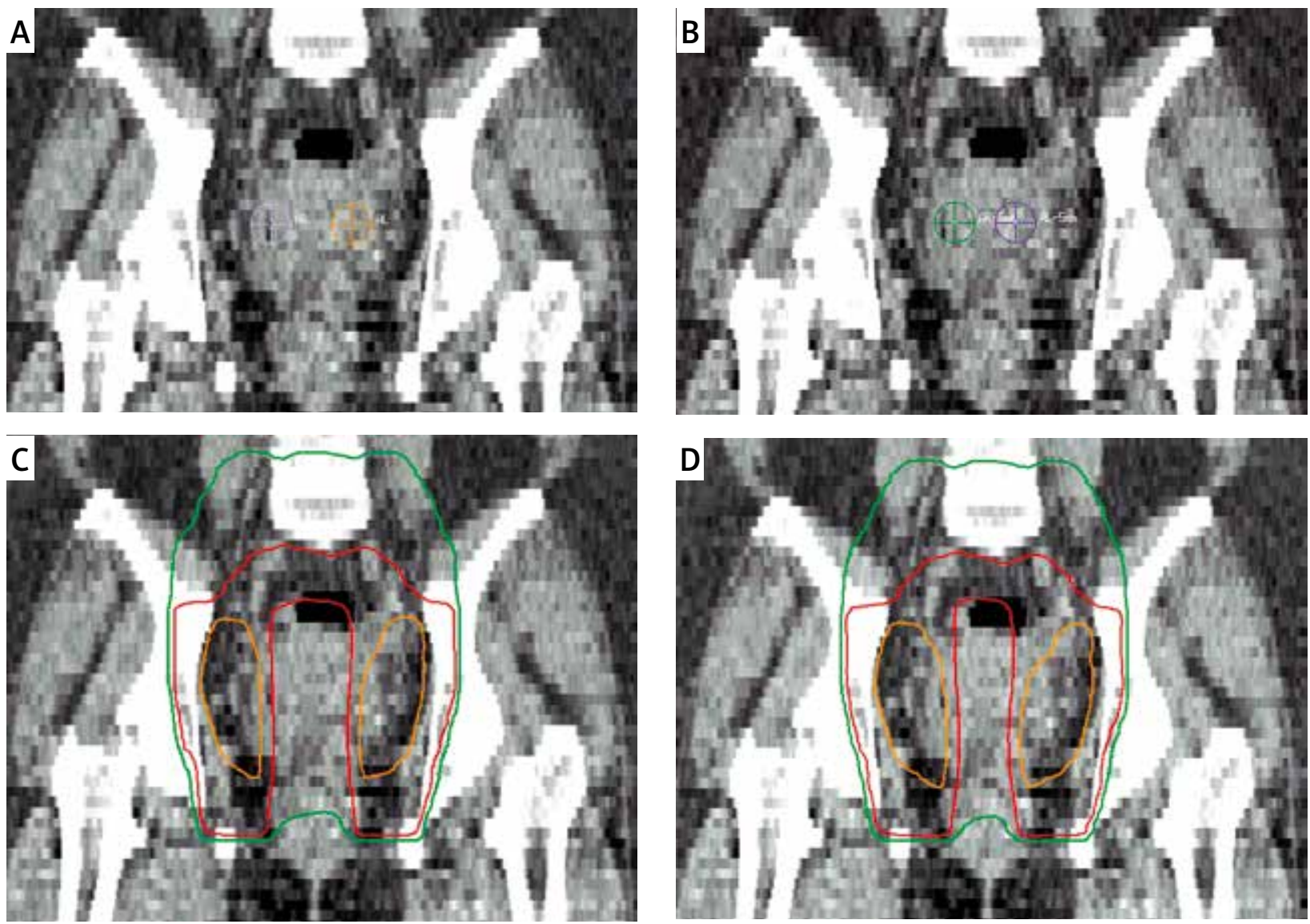

Fig. 1. Coronal CT images showed AR/AL (A), AR-5mm/AL-5mm (B), isodose of 4-cm central shielding (C), and isodose of 3 -cm central shielding (D) (note: $44 \mathrm{~Gy}=$ green, $50 \mathrm{~Gy}=$ red and $56 \mathrm{~Gy}=$ orange) 


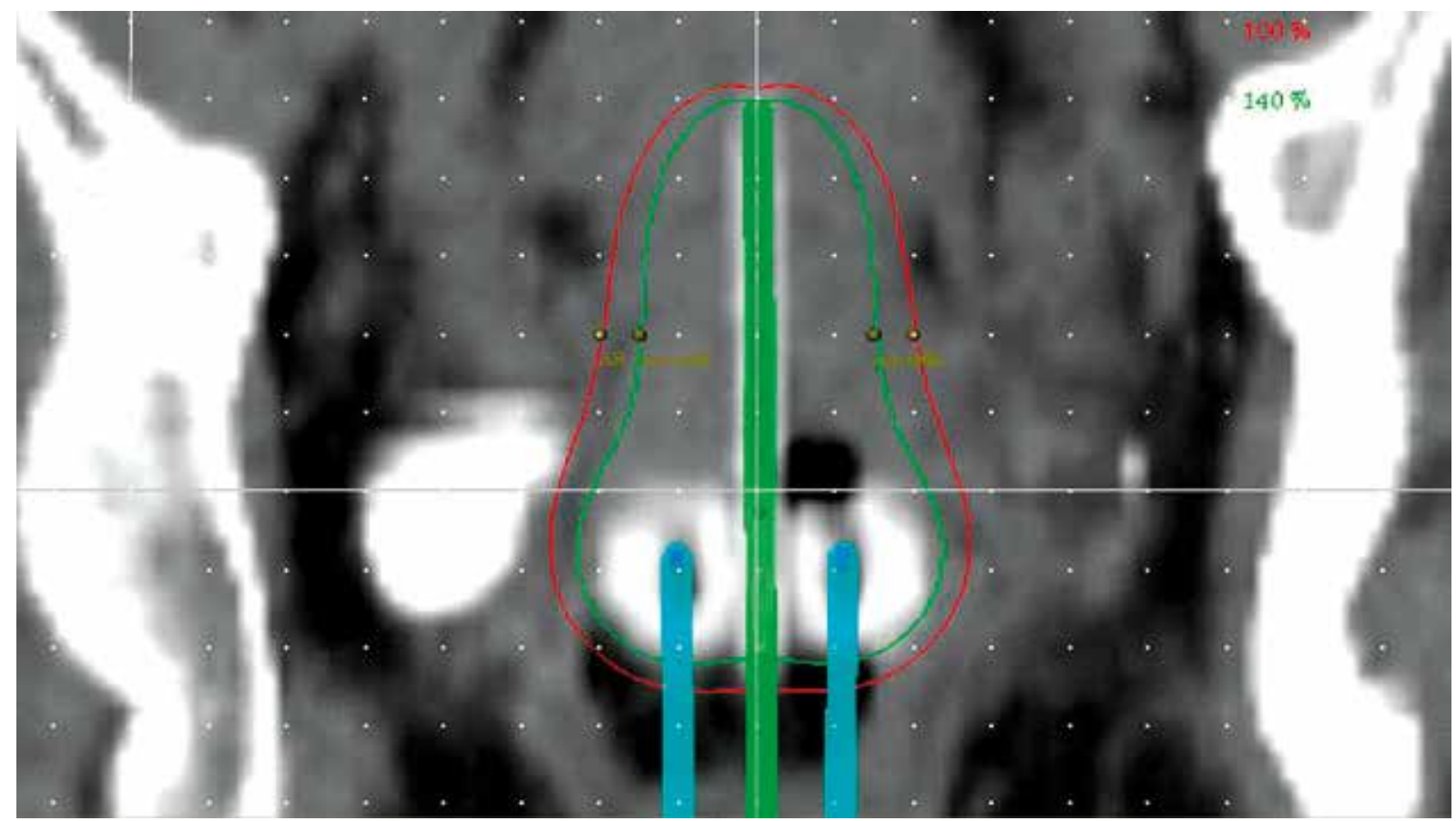

Fig. 2. HDR brachytherapy in conventional loading showed point A and point A-5mm

Table 1. Physical dose at point A and A-5mm for 3-cm and 4-cm central shielding

\begin{tabular}{lcccc} 
3-cm CS & $\begin{array}{c}\text { Phase I } \\
(22 f)\end{array}$ & $\begin{array}{c}\text { Phase II } \\
(3 f)\end{array}$ & $\begin{array}{c}\text { Phase III } \\
(3 f)\end{array}$ & Total \\
\hline AR & 45.4 & 5.4 & 5.2 & 56 \\
\hline AL & 45.2 & 5.4 & 5.2 & 55.8 \\
\hline AR-5mm & 45.1 & 3.2 & 3.1 & 51.4 \\
\hline AL-5mm & 44.9 & 3.4 & 3.3 & 51.6 \\
4-cm CS & $\begin{array}{c}\text { Phase I } \\
(22 f)\end{array}$ & $\begin{array}{c}\text { Phase II } \\
(3 f)\end{array}$ & $\begin{array}{c}\text { Phase III } \\
(3 f)\end{array}$ & Total \\
\hline AR & 45.4 & 3.1 & 2.9 & 51.4 \\
\hline AL & 45.2 & 3.4 & 3.2 & 51.8 \\
\hline AR-5mm & 45.1 & 1.3 & 1.1 & 47.5 \\
\hline AL-5mm & 44.9 & 1.4 & 1.2 & 47.5 \\
\hline
\end{tabular}

it caused a higher (about $4 \mathrm{~Gy}$ ) cumulative dose in EQD2 $(\alpha / \beta=10)$ at point $A$ than $4 \mathrm{~cm}$ central shielding. These findings have a few points of concern. Firstly, this situation occurred in good geometry situation where point A and A-5mm were added at the same point for WPRT and brachytherapy. However, the issue regarding uncertainties considering the interfraction variations during WPRT and brachytherapy phases, caused the difficulties for evaluation. Secondly, this purpose aimed to evaluate the cumulative dose to point A in EQD2 era when we applied the different size of central shielding for conventionally point-based planning. With the variation of tumor size of each patient, it could be problematic to evaluate practically. If the tumor is greater than the shielding, it will cause the higher and lower areas in tumor. On the contrary, if the tumor is smaller than the shielding, it might
Table 2. Dose at point $A$ and $A-5 m m$ for $3-\mathrm{cm}$ and 4-cm central shielding in EQD2 concepts

\begin{tabular}{lcccc}
$3 \mathrm{~cm}$ & $\begin{array}{c}\text { Phase I } \\
(22 \mathrm{f})\end{array}$ & $\begin{array}{c}\text { Phase II } \\
(3 \mathrm{f})\end{array}$ & $\begin{array}{c}\text { Phase III } \\
(3 \mathrm{f})\end{array}$ & Total \\
\hline AR & 45.6 & 5.3 & 5.1 & 56.0 \\
\hline $\mathrm{AL}$ & 45.4 & 5.3 & 5.1 & 55.8 \\
\hline AR-5mm & 45.3 & 3.0 & 2.9 & 51.1 \\
\hline AL-5mm & 45.1 & 3.2 & 3.1 & 51.3 \\
$4 \mathrm{~cm}$ & $\begin{array}{c}\text { Phase I } \\
(22 f)\end{array}$ & $\begin{array}{c}\text { Phase II } \\
(3 \mathrm{f})\end{array}$ & $\begin{array}{c}\text { Phase III } \\
(3 \mathrm{f})\end{array}$ & Total \\
\hline AR & 45.6 & 2.9 & 2.7 & 51.1 \\
\hline AL & 45.4 & 3.2 & 3.0 & 51.5 \\
\hline AR-5mm & 45.3 & 1.1 & 1.0 & 47.4 \\
\hline AL-5mm & 45.1 & 1.2 & 1.0 & 47.3 \\
\hline
\end{tabular}

Table 3. Cumulative doses in EQD2 concepts

\begin{tabular}{lcc} 
Parameters & 3-cm shielding & 4-cm shielding \\
\hline AR & 95.7 & 90.8 \\
\hline AL & 95.5 & 91.2 \\
\hline AR-5mm & 115.8 & 112.0 \\
\hline AL-5mm & 115.9 & 112.0 \\
\hline
\end{tabular}

receive very high dose from brachytherapy. To solve this problem, the central shielding can be tailored according to the clinical target volume (HR-CTV) and GEC-ESTRO recommendations, plus treatment with image guided brachytherapy to get the accurate dose to HR-CTV $[9,10]$. This approach will improve the calculation of cumulative 
dose in EQD2 to the HR-CTV in the treatment with central shielding.

\section{Conclusions}

With the different central shielding, our calculations showed a variance between $3 \mathrm{~cm}$ and $4 \mathrm{~cm}$ central shielding in terms of EQD2 in point A. In order to solve this problem, the central shielding can be tailored according to the HR-CTV from image-guided brachytherapy.

\section{Acknowledgement}

The author offers many thanks to the staff of the Division of Therapeutic Radiology and Oncology, Faculty of Medicine, Chiang Mai University for supporting this study.

\section{Disclosure}

Authors report no conflict of interest.

\section{References}

1. Kamnerdsupaphon P, Srisukho S, Sumitsawan Y et al. Cancers in Northern Thailand. Biomed Imaging Interv J 2008; 4: e46.

2. Rose PG, Bundy BN, Watkins EB et al. Concurrent cisplatin-based radiotherapy and chemotherapy for locally advanced cervical cancer. N Engl J Med 1999; 340: 1144-1153.

3. Keys HM, Bundy BN, Stehman FB et al. Cisplatin, radiation, and adjuvant hysterectomy compared with radiation and adjuvant hysterectomy for bulky stage IB cervical carcinoma. N Engl J Med 1999; 340: 1154-1161.

4. Eifel PJ, Winter K, Morris $\mathrm{M}$ et al. Pelvic irradiation with con-current chemotherapy versus pelvic and para-aortic irradiation for high-risk cervical cancer: An update of Radiation Therapy Oncology Group trial (RTOG) 90-01. J Clin Oncol 2004; 22: 872-880.

5. Lorvidhaya V, Chitapanarux I, Sangruchi S et al. Concurrent mitomycin C, 5-fluorouracil, and radiotherapy in the treatment of locally advanced carcinoma of the cervix: a randomized trial. Int J Radiat Oncol Biol Phys 2003; 55: 1226-1232.

6. Bentzen SM, Joiner MC. The linear-quadratic approach in clinical practice. In: Basic clinical radiobiology. Joiner M, Van Der Kogel A (eds.). $4^{\text {th }}$ ed. Edward Arnold, London 2009; pp. 120-134.

7. Nag S, Erickson B, Thomadsen B et al. The American Brachytherapy Society recommendations for high-dose-rate brachytherapy for carcinoma of the cervix. Int J Radiat Oncol Biol Phys 2000; 48: 201-211.

8. Nakano T, Kato S, Ohno T et al. Long-term results of highdose rate intracavitary brachytherapy for squamous cell carcinoma of the uterine cervix. Cancer 2005; 103: 92-101.

9. Haie-Meder C, Pötter R, VanLimbergen E et al. Recommendations from gynaecological (GYN) GEC-ESTRO working group (I): concepts and terms in 3D image based 3D treatment planning in cervix cancer brachytherapy with emphasis on MRI assessment of GTV and CTV. Radiother Oncol 2005; 74: 235-245.

10. Pötter R, Haie-Meder C, Van Limbergen E et al. Recommendations from gynaecological (GYN) GEC ESTRO Working Group (II): Concepts and terms in 3D image-based treatment planning in cervix cancer brachytherapy-3D dose volume parameters and aspects of 3D image-based anatomy, radiation physics, radiobiology. Radiother Oncol 2006; 78: 67-77. 